\title{
The Medical \& Health Maintenance Tourism and Ecosystem of Industries around TCM Services
}

\author{
Mujiexin $\mathrm{Liu}^{1.2}$, Min $\mathrm{Liu}^{1.23^{*}}$, Dazheng Zhang ${ }^{1.3 .4}$, Zhidong Jia ${ }^{1.5}$ \\ 'Qingcheng Institute of Medicine, Dujiangyan, Chengdu, Sichuan, China \\ ${ }^{2}$ Chengdu University of Traditional Chinese Medicine, Chengdu, Sichuan, China \\ ${ }^{3}$ Dujiangyan Medical Centre, Dujiangyan, Chengdu, Sichuan, China \\ ${ }^{4}$ No.5 People's Hospital of Dujiangyan, Qingchengshan, Dujiangyan, Chengdu, Sichuan, China \\ ${ }^{5}$ Chengdu Neusoft University, Qingchengshan, Dujiangyan, Chengdu, Sichuan, China
}

\begin{abstract}
Background: Medical and health maintenance tourism (MHMT) is rising fast in our aging society with the largest population in the world. At the very core of its development, medical services are crucial for driving the development of the related industries that can develop into an ecosystem for sustainability. Methods: Through the market analysis, it is made very clear that the diversified demands can only be met by the medical services around TCM with its various advantages, which are proven in the example of the success of Mt. Qingcheng as Tianfu Qingcheng Resort for MHMT. Results: With TCM services as the driving force, the local hospitals, tertiary industry, transportation and logistics, and the R\&D of new medicine grow into an ecosystem. Conclusions: The green ecosystem will enable the sustainable development of MHMT.
\end{abstract}

\section{MHMT market analysis}

The market for medical and health maintenance tourism (MHMT) has been growing rapidly among many groups of people. According to the Statistical Communique of the People's Republic of China on the Development of Health Undertaking in 2018 issued by the Department of Planning and Information of the National Health Commission, the average life expectancy (ALE) of Chinese population was 77.0 years in 2018. [1] Thanks to the improving quality of life (QOL), the healthy, and independent elderly tourists will continue to increase, which further contributes to elderly tourism. [2] A great number of independent elderly tourists can afford medical and health maintenance (MHM) tours both physically and economically, though China is the world's largest aging society with people aged 65 years and above over 158 million by the end of 2017, which is expected to expand to 366 million in 2050. [3,4] These people can be divided by their demands into different groups: the relatively healthy ones who travel to places for health maintenance, those with ailments pay visits to specific doctors or hospitals, and those with chronical disease need to stay in an agreeable environment with professional medical services, and those who are near their retirement age with greater demand for MHMT because they suffer from and are quite aware of their health problems. They are becoming the major consumers of MHMT though time doesn't allow them to stay in a place for long. While young people, who travel as their purpose, may still try therapeutic massage and medical beauty services apart from consuming medical products. Facing such huge and diverse demands, hospitals can only play a very small role. And it is never enough for destinations just to be located by the sea, in the forest, or with hot springs, such as Bama [5], southern coastal areas and Hainan. To attract MHM tourists, the capabilities of providing services for medical treatment and maintenance are as important as the nice weather and wonderful environment. Only TCM has the capabilities not only to provide medical services to diverse consumers but also drive the development of related industries into an ecosystem.

\section{The advantages of TCM in MHMT}

With the largest population and the improving living standard in China, the number of MHM tourists will continue to grow. Apart from the diversified demands of the market, new diseases and complications brought about by the chronicle diseases are far beyond the science of western medicine. Take COVID 19, for example. Ventilators can only make the patients even more painful without a certain positive outcome, while TCM can handle this disease, a type of flu, easily with the traditional medicine passed down by our ancestors to discharge the virus without any unwanted side-effects $[6,7]$, no matter how novel the viruses can be. And the complications of Type II diabetes (T2D), various and severe, which many people suffer from in their daily life, are highly challenging to the world of western medicine $[8,9,10,11]$. But in TCM, all complications are clearly recorded in the books of ShangHanLun and JinKuiYaoLue by Zhang Zhongjing (about 150-154 A.D to 215-219 A.D.), which

"Corresponding author: liumin0109@126.com 
all takes time to treat and maintain with different prescriptions at different times and conditions [12, 13]. The precision of diagnosis and the level of individualized treatment that TCM can provide are far beyond the knowledge of western medicine. This is why the government became so determined to push the development of TCM. It is required that all students majored in western medicine must learn TCM to complete their medical degrees $[14,15]$. And it is impossible for all the patients of with chronical diseases to stay in hospitals while all kinds of complications may occur and become severe in their everyday life. Take the heart problems of T2D patients as an example. With the prescriptions from the ancient classis and the management through the right food structure with specific intake amount and order, the very severe conditions of heart troubles can be handled with just two doses of one prescription for enhancing the innate capability of the spleen and stomach to produce and move the blood, while reducing the heat already accumulated in the chest. For those still at work or in the condition of suboptimal health status (SHS), they have no time to stay in the hospital and doctors have no way to change their unhealthy lifestyles $[16,17,18]$. On the contrary, it doesn't need to keep anyone in the hospital when the right prescription is given and the medicine is taken at home, which is true for the treatment for all diseases by TCM. For young and healthy people on the tour, therapeutic massages, acupunctures and skincare through enhancing and balancing the functionalities of internal organs with traditional medicine under the supervision of TCM doctors. Most traditional medicines can largely revive the strength and energy without causing any danger and damages to the body $[19,20,21,22]$. The needed training for certified professionals to meet the demands can only be carried out by TCM doctors.

The efficiency of TCM cannot be matched by western medicine when one TCM doctor can be considered as a whole hospital [23]. Facing large amounts of people scattered outside the hospital, the mobility of TCM doctors can provide the needed and individualized services in time without the need of using those heavy and expensive machines for diagnosis and prescription. It is cost-effective both to the medical institutions and the consumers. What's more, the most basic TCM principle and practice of prevention and early intervention not only just resonate with the growing market demand for MHMT, but largely reduce the burden brought about by diseases both to the individuals and the medical system. When this aspect is considered in MHMT, its long-lasting costefficiency and high effectiveness in practice are the biggest attractions.

\section{The resources-integration for MHMT around TCM services - Mt. Qingcheng as an example}

Mt. Qingcheng, a renowned AAAAA tourist resort of longevity with the best quality of air and water from the $59.27 \%$ forest coverage, has long been a destination for health maintenance and post-operative rehabilitation not only because of its very rich natural resources but also the development of health industries pushed by TCM services. [24]

Triggered by the national standards of classifying tourist's resorts issued in 2015, Dujiangyan municipal government kicked off the work on establishing a tourist resort at the national level targeting to make $\mathrm{Mt}$ Qingcheng a model of MHMT destination at the world level. [25] A provincial program applied together by Qingcheng Institute of TCM and Chengdu Neusoft University, The Research \& Development and Application Models of Comprehensive Service Platform for Smart Health Tourism in Dujiangyan (2020-YF09- 00010-SN), was approved in June, 2020. The research team collected and sorted out all the information related to tourism resorts, hospitality industry, medical service institutions, transportation and logistics. To effectively support the industries related to MHMT, a comprehensive service platform was designed and is being built around TCM services tailored for the market demands of MHMT, which are classified into four categories: health management, visits to doctors' offices, treatment \& rehabilitation, and distant diagnosis.

The hospitals, including Level III Category A hospitals in this area like Dujiangyan Medical Centre, Dujiangyan Health Centre for Women \& Children and Dujiangyan Hospital of TCM, Level II Category A hospital like Dujiangyan No 1. People's Hospital, 7 Level II Category B \& C clinics and 6 other smaller hospitals and medical institutions, are all integrated into the platform. Qingcheng Institute of Medicine and the TCM postdoctoral workshop at the foot of Mt. Qingcheng shouldered the organization and integration of TCM resources in the area of Chengdu [26]. The high mobility of TCM doctors and online services make it possible to provide services within $15 \mathrm{~m}$ in the area of Mt. Qingcheng.

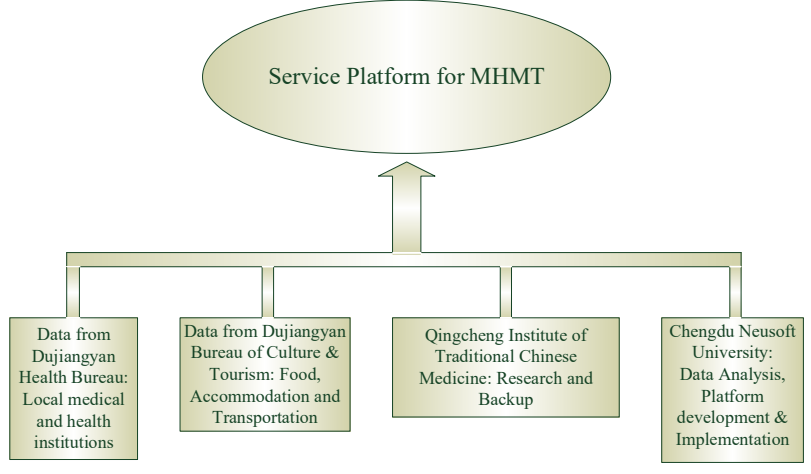

The Support Platform for MHMT

Fig 1. The Support Platform for MHMT.

To maximize the services, the local hospitality industry is highly involved in the process of MHMT by providing lodging and accommodation through the promotion of the local TCM services. For example, the local hotels and inns can help patients register for their desired doctors while providing services including food \& accommodation, transportation to and from hospitals, and the preparation of the traditional medicine and the food of the required recipes for recovery. To cope with the growing demands brought about by the TCM services for different MHM tourists: those who have chronical 
diseases, those who have to stay for post-operative rehabilitation, those who come just for specific experts for their diseases, and those who come here just to enjoy the cool summer for the wellbeing, the government has been making great efforts to build the network of transportation including railways, highways, M-TR, buses apart from airports under construction. Even after tourist leave this place, distant-service packages extend the coverage and duration of TCM services.

In May 19, 2019, the National Ministry of Culture and Tourism officially recognized the areas of Mt. Qingcheng as Tianfu Qingcheng Resort for MHMT [27, 28]. The well integration of healthcare and tourism around TCM services has largely boosted the development of the related industries and will be the engine for the future development.

\section{The Driving force of the TCM services for the development}

For MHMT purposes, people not just travel to their destinations. It is more about staying in a place to enjoy the high quality of medical services in the desirable weather and natural conditions for the recovery. Only when it is possible, like TCM does, to provide the diversified and tailored services for great number of people scattered in an area with different health conditions, can their stays last long enough from weeks to months, to repeated weekly and annual MHMT. Naturally, the local hospitals, tertiary industry, transportation \& logistics, and the R\&D of new medicine all continue to flourish to meet the market demands.

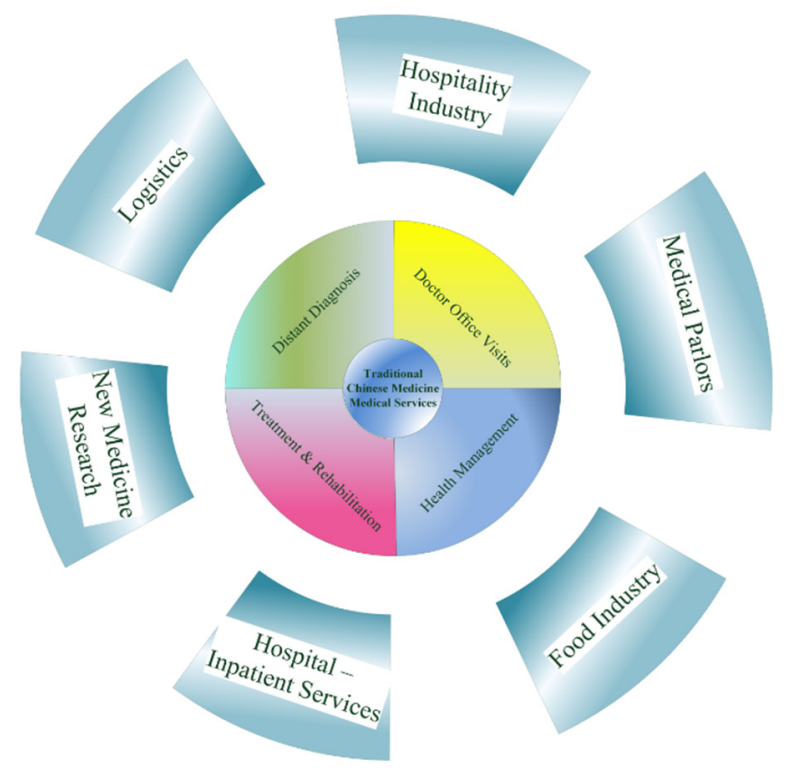

The MHMT Eco-System Driven by TCM

Fig 2. The MHMT Eco-System Driven by TCM

For very serious inpatients in the hospitals, TCM is even more important for their outcome rather than just the high-level facilities and machines, because there are solutions from TCM perspective for all complications. Together with the desirable natural environment, high level of facilities and machines at the hospitals, people may consider to travel for a more satisfied outcome. In the past, only the rich few could afford the expenses for long stays in the hospitals. To meet the increasing demand, new hospitals have to be built while the old ones have to be upgraded. But for most people with chronical diseases who come for rehabilitation, they need to rent places or stay at the local B\&Bs for the needed services that can be provided by TCM outside the hospitals. Many people even buy houses in such areas for retirement to enjoy the local natural environment and medical services. To house these different groups of people for MHMT purposes, the local real-estate and rentals have to grow fast to meet the market demands in numbers and varieties.

Along the upheaving sales and the rentals of local housing, communities that are suitable for independent elderlies are at high demands for longer stays and even serve as seasonal senior homes. In the area of Mt. Qingcheng, for example, some hotels provide suites with kitchen and washing machines so that people can live in this place for longer time rather than just weekend stays. Some local communities for MHMT purposes provide the facilities and support for the maintenance of physical functionalities with the guidance of TCM doctors.

As MHMT is more about improving health conditions, TCM services like therapeutic massage, acupuncture and moxibustion with Artemisia argyi can be widely carried out through training. The certified professionals can start their own business as these services are highly welcome in China and far exceed the capabilities of hospitals. Many people need long-term therapeutic massages after the visits to doctors or hospitals. Guided by the TCM prescriptions and supervised by TCM doctors, these professionals are so mobile that they can extend the zone of service and number of customers. Similarly, TCM beauty and spa parlors are no longer just places for care and massage, but can also provide individualized and customized TCM prescriptions for the real improvement of skin and hair conditions induced by the improved functionalities of the internal organs, which can greatly increase the quality and generate high returns for the business owners.

Eating is the highest priority in everyday life in China. Nutritious food based on TCM prescriptions is crucial for recovery and post-operative rehabilitations. In TCM, many ingredients of food are medicine themselves and many herbs can be used as ingredients for cooking. But without the TCM knowledge about the attributes and the consequence of the interactions of different herbs and food, it can be very harmful to health. Thus, supported by TCM doctors, customized cuisines effectively help improve the health conditions of the customers. General recipes for different seasons have been an attraction in many places like the area of Mt. Qingcheng for decades. The food industry will not only develop fast, but eventually help build a unique food culture.

Besides, with the large numbers of MHM tourists and their medical data, the research and development of medicine can become much more cost-effective. To 
support the fast development of these industries, the transportation and logistics have to be in pace so that an ecosystem can become sustainable for the future development of MHMT.

\section{Conclusion}

MHMT, not an independent industry itself, is the engine for the development of an ecosystem boosting the tertiary industry, real-estate, transportation and logistics. Being very green and at this time of our aging society of the largest population in the world, MHMT is very promising in the near future with TCM services as the major driving force for the full-ledged development of the health industry.

\section{Funding:}

1. The development and application demonstration of the comprehensive service platform for health maintenance and tour in Dujiangyan, Chengdu Science and Technology Bureau, 2020-YF09-00010-SN

2. Research on the ecosystem of the health industry with traditional Chinese medicine as the core, Sichuan Provincial Department of Education, Xq20C03.

\section{References}

1. Gui, S., \& Chen, J. (2019). Average life expectancy of the Chinese population in 1949-2019: trends, contributors and prospects. China Population and Development Studies. doi:10.1007/s42379-01900036-3

2. Martin, L. G., \& Preston, S. H. (1994). Demography of aging. Washington D.C.: National Academy Press; Losada, N., Alén, E., Cotos-Yáñez, T., \& Domínguez, T. (2019). Spatial heterogeneity in Spain for senior travel behavior. Tourism Management, 70, 444-452

3. National Bureau of Statistics of the People's Republic of China. 2018 China Statistical Yearbook. http://www.stats.gov.cn/tjsj/ndsj/2018/indexeh.htm. Accessed June 27, 2019. [in Chinese]

4. The Population Division of the Department of Economic and Social Affairs of United Nations Secretariat. World Population Prospects: The 2019 Revision. https://population.un.org/wpp/. Accessed June 27, 2019

5. Wang, K., Xu, H., \& Huang, L. (2020). Wellness tourism and spatial stigma: A case study of Bama, China. Tourism Management, 78, 104039. doi: 10.1016/j.tourman.2019.104039

6. Zhang, Kai. "Is traditional Chinese medicine useful in the treatment of COVID-19?" The American journal of emergency medicine 38.10 (2020): 2238

7. Zhao, Zhenyu, et al. "Prevention and treatment of COVID-19 using Traditional Chinese Medicine: A review." Phytomedicine (2020): 153308.

8. Solini, Anna, and Ivana Novak. "Role of the P2X7 receptor in the pathogenesis of type 2 diabetes and its microvascular complications." Current opinion in pharmacology 47 (2019): 75-81

9. Gabryelska, Agata, et al. "HIF- $1 \alpha$ as a mediator of insulin resistance, T2DM, and its complications: potential links with obstructive sleep apnea." Frontiers in Physiology 11 (2020)

10. Halim, Michael, and Alice Halim. "The effects of inflammation, aging and oxidative stress on the pathogenesis of diabetes mellitus (type 2 diabetes)." Diabetes \& Metabolic Syndrome: Clinical Research \& Reviews 13.2 (2019): 1165-1172

11. Mahdi, Ali, et al. "Red blood cell peroxynitrite causes endothelial dysfunction in type 2 diabetes mellitus via arginase." Cells 9.7 (2020): 1712.

12. Mitchell, Craig, et al. "Shang Han Lun: On cold damage, translation \& commentaries." (1998)

13. Jing, Zhang Zhong. "Jin Kui Yao Lue Fang Lun." (1987).

14. Policy Measures on Accelerating the Development of Specialized TCM (2021/01/22), Notice on Policy Measures on Accelerating Specialized TCM issued by the State Council (Office of the State Council (2021) No.3) [In Chinese] (www.gov.cn)

15. The $13^{\text {th }}$ Five-Year Plan on the Further Reform of the Medical and Health System (2016) issued by the State Council [In Chinese] (www.gov.cn)

16. Chen, Jieyu, et al. "The role of healthy lifestyle in the implementation of regressing suboptimal health status among college students in China: a nested casecontrol study." International journal of environmental research and public health 14.3 (2017): 240

17. Wang, Youxin, et al. "Association between ideal cardiovascular health metrics and suboptimal health status in Chinese population." Scientific reports 7.1 (2017): 1-6

18. Ding, Guoyong, et al. "Evaluation of the relationship between cognitive impairment and suboptimal health status in a northern Chinese population: a crosssectional study." Journal of global health 10.1 (2020).

19. Cherkin, Daniel C., et al. "Randomized trial comparing traditional Chinese medical acupuncture, therapeutic massage, and self-care education for chronic low back pain." Archives of internal medicine 161.8 (2001): 1081-1088

20. Irnich, Dominik, et al. "Randomized trial of acupuncture compared with conventional massage and "sham" laser acupuncture for treatment of chronic neck pain Commentary: Controls for acupuncture - can we finally see the light?" Bmj 322.7302 (2001): 1574

21. Shiyi, W. A. N. G., et al. "Innovation and inheritance: A novel strategy for the development of antiyellowing cosmetic material from TCM formula." (2020)

22. Qi, Lu. "The Application of the" Prevention Diseases" of TCM's Concept to cosmetic dermatology." Chinese Journal of Aesthetic Medicine 10 (2008); Goldstein, Shellie. "Cosmetic 
Acupuncture: Clues to Overall Health."

23. Koo, John, and Sumaira Arain. "Traditional Chinese medicine for the treatment of dermatologic disorders." Archives of Dermatology 134.11 (1998): 1388-1393

24. Liu, Mujiexin, Min Liu, and Dazheng Zhang. "Developing TCM-Centered Ecology of Health Care and Maintenance Industries--With the Health Ecosystem around Mt. Qingcheng as an Example." 2020 International Conference on Advanced Education, Management and Information Technology (AEMIT 2020). Atlantis Press, 2020

25. Sichuan Culture and Tourism Bureau. http://wlt.sc.gov.cn/scwlt/cyly/2019/5/19/d69feb27f a9b409c9cec358dfb9a5ec4.shtml

26. Liu, Mujiexin, Min Liu, and Dazheng Zhang. "Qingcheng TCM Medical Treatment and Health Maintenance Model-O2O Based." 5th International Conference on Social Sciences and Economic Development (ICSSED 2020). Atlantis Press, 2020.

27. The Publicity of the Name List of the Tourist Resorts at the National Level issued by the Ministry of Culture

and Tourismhttp://zwgk.mct.gov.cn/zfxxgkml/zykf/2020 12/t20201206 917344.html [In Chinese]

28. The Classification of the Tourist Resorts (GBT263582010) http://www.gb688.cn/bzgk/gb/newGbInfo?hcno=15 F06C554FFC782E19DA4915ECF6CCBB Chinese] 